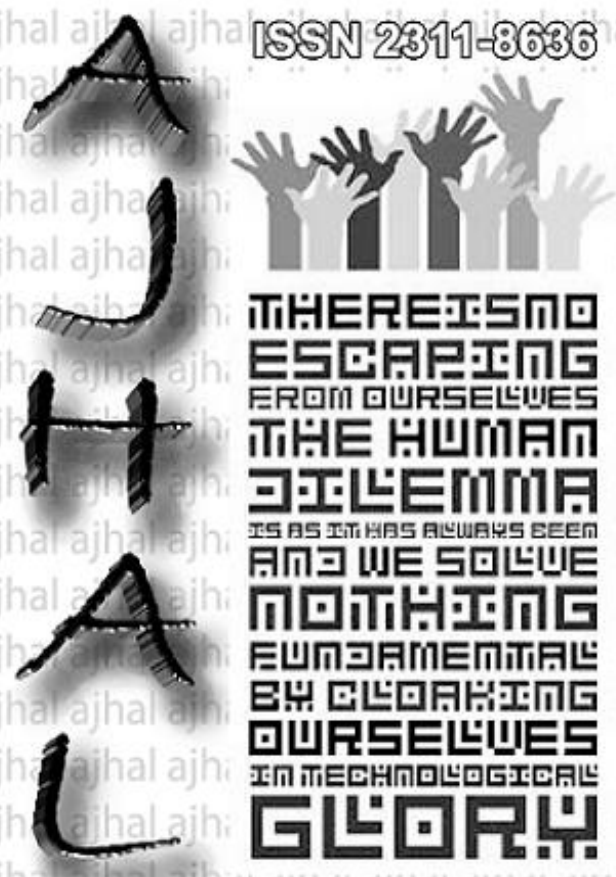

Vol 3, No. 2/2016

Asjan Jourmal of Humanity, Art and Literatulue 


\section{Blockchain as a Type of Distributed Ledger Technology}

\section{Koteswara Rao Ballamudi}

ISSN: 2311-8636 (Print)

ISSN: 2312-2021 (Online)

DOI prefix: 10.18034

Licensed:

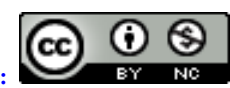

Source of Support: Nil

Conflict of Interest: None Declared

${ }^{*}$ Email for correspondence:

koteswarabv@gmail.com
Solution Architect, Software Engineering Architecture Department, Cyber Global, Inc., USA

\section{ABSTRACT}

Distributed ledger technology (DLT) that stores data (usually immutable and sequenced transaction records) in a decentralized way through cryptography and consensus algorithms. The first widely recognized implementation of the blockchain took place in 2009 on the Bitcoin public blockchain. Since then, other types of blockchain have been developed for a wide range of applications and features built on common principles such as decentralization, encryption, consensus, and immutability. In particular, blockchain technology is most widely used in transaction settlement and digital currency banks and the financial sector, as well as in supply chain applications that help participants solve problems quickly and efficiently. Other use cases continue to be developed. As a form of information management, blockchain and related DLTs offer advantages over traditional databases and may help develop certain new technologies such as the Internet of Things. Blockchain regulation is currently restricted at the international and federal levels, but state-level legislation provides support and awareness of aspects of blockchain technology. Most of the current regulations are in the form of selfregulation by blockchain developers and related communities, but many challenges and risks such as data privacy and security need to be addressed in the near future.

Key Words: Blockchain, DTL, Cryptography

\section{INTRODUCTION}

Blockchains come in many forms and generally share four key functions. Distributed data storage (public ledger of transaction records), encryption, immutability, and consensus algorithms. As a special type of distributed ledger technology (DLT), blockchain stores encrypted data throughout a peer-to-peer network, linking contiguous "blocks" of information into "chains." Information available to all network participants is all information transactions on the blockchain. The consensus algorithm ensures that the information is consistently immutable throughout this distributed network and prevents individual users from adding it to the ledger information without permission from the network. In addition, the structure of the blockchain does not allow you to edit or delete previous information about the chain. Editing will break the integrity of the distributed ledger. 
One of the common blockchain classification schemes is about membership or accessibility. The public blockchain has a publicly visible ledger and is fully decentralized. The user may need to allow writes or additions to the blockchain. Most cryptocurrencies are implemented as public blockchains. The private blockchain is accessible only to specific host organizations and is primarily used for internal purposes such as auditing and record management. The consortium blockchain (or hybrid blockchain) is semiprivate and accessible to a range of organizational groups such as banks, energy traders and hospitals, facilitating more efficient transactions or information sharing between the parties. The type of blockchain depends on its purpose and can be a different design schema. Private and consortium blockchains are less decentralized and focus on smaller user groups. Therefore, they can be more sustainable and cost effective than public blockchain.

Within the public blockchain, there are various blockchain implementations with different design schemas between attributes such as consensus algorithms, data accessibility, variability, decentralization, and design architecture focus. In addition, some blockchain projects focus on developing the blockchain architecture itself (that is, at the platform level), while other blockchain projects are for implementing specific functionality (that is, application level). Is built on top of the blockchain.

In addition to blockchain, other forms of DLT include hash graphs, directed acyclic graphs, and tangles. Hash graphs are DLTs that rely on computational gossip to achieve consensus and are structured like an interwoven chain. A node-to-node transaction to implement a new network structure that is optimal for Internet of Things (IoT) applications.

\section{Common Applications and Market DeVelopment}

As a form of information management, blockchain can and has been applied to many industries, from IT to healthcare and energy. The blockchain market has grown rapidly over the last three years, with key companies in this area divided into three groups that directly reflect the three blockchain categories: public, private and consortium.

Most public blockchains offer Platform-as-a-Service (PaaS), which provides tools for users to develop blockchain-based applications or DApps (distributed applications). Such DApps are at various stages of market preparation, and the most popular deployed DApps include those focused on games, trading, and payments. Cryptocurrencies are also implemented in public blockchains and usually exist as coins (similar to digital currencies), utility tokens (tokens used as blockchain royalties), or security tokens (tokens representing digital security). To do. Of the three blockchain types, public blockchain has fueled many of the recent blockchain hype and initial coin offerings (ICOs), which can pose significant legal and regulatory risks. In particular, many "users" of public blockchain use them as speculative investments (ie cryptocurrencies) rather than blockchain technology itself. Key groups working on the public blockchain include Ripple Labs (Ripple Protocol), Ethereum Foundation (Ethereum Public Chain), and block.one (EOS Public Chain).

Private blockchains tend to be offered as Software-as-a-Service (SaaS) or Infrastructure-asa-Service (IaaS), with general (such as blockchain network management) or industryspecific features (electronic). For voting and supply chain tracking). Most enterprise solutions and applications are built on a private blockchain. It is more practical than public 
blockchain because it reduces energy costs, improves consensus efficiency, reduces processing time, and enhances privacy. Many private blockchains are hard forks of public blockchains or are inspired by public blockchains. The main groups include Amazon (Amazon Managed Blockchain), IBM (IBM Blockchain), Microsoft (Azure Blockchain Workbench), MasterCard (MasterCard Blockchain API), and JP Morgan (Quorum).

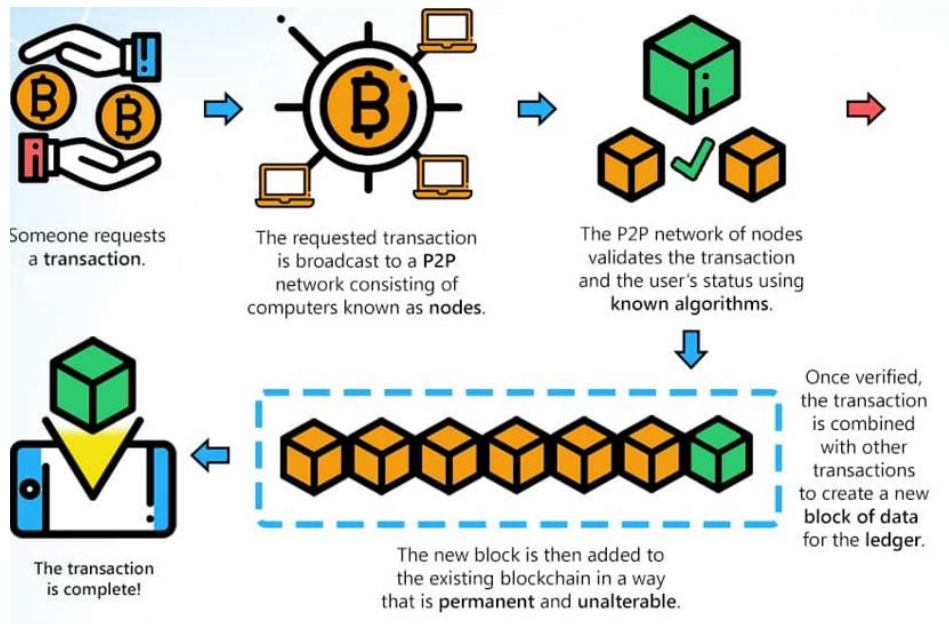

Blockgeeks

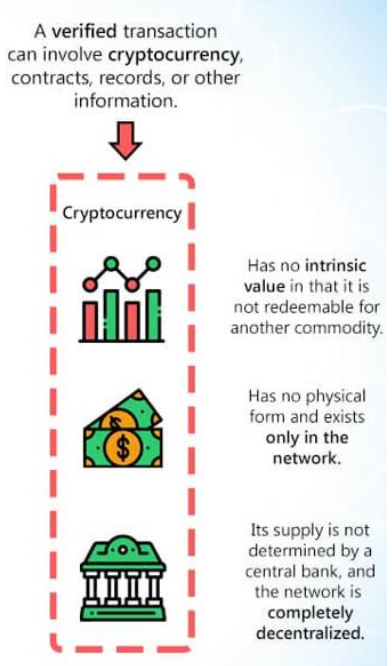

\section{Public Purpose Considerations}

Blockchain has the potential to advance important elements of public purpose, such as:

- Data security: Blockchain can enhance data security by decentralizing or storing and updating an exact copy of the same ledger on all system nodes at the same time. This prevents individual actors from controlling or maliciously editing the data ledger, and when combined with encryption, offers superior advantages over centralized databases.

- Data integrity: The blockchain, along with the consensus algorithm and private key identifier, ensures data integrity and verifies that all stored data is approved by the consensus algorithm (ie, external). No unnecessary data has been inserted). Depending on the type of data and architecture, the blockchain may also verify the reliability of the data and the validity of the transaction.

- Transparency: All transactions and information on the blockchain are available on the network in encrypted form. This allows you to increase the transparency of real-world processes and information such as IDs, electronic voting, and utility bills.

- Comprehensive / Accessibility: Blockchain helps connect local people to digital infrastructure in a secure way across different domains, including microfinance, access to accurate market prices and information, and verification of humanitarian aid.

\section{Blockchain Faces Risks and Challenges}

- Data privacy and ownership: Data privacy and ownership is one of the biggest challenges for blockchain, as blockchain information is decentralized and immutable. Some blockchain features appear to be inconsistent with existing regulations (such as 
right to be forgotten and blockchain data persistence), while other data privacy features such as identity verification are across the blockchain. Not implemented consistently.

- Legality: Since smart contracts are one of the popular tools in blockchain, the validity and legality of smart contracts and other blockchain-based contracts must be consistent with traditional contract law principles. There are 27. In addition, blockchain provides tools to transfer digital ownership Assets and information, and therefore effective rules and governance structures to regulate the transfer of ownership are important.

- Financial Risk: Cryptocurrencies and tokens are currently one of the main uses of blockchain and require additional certainty in this area (such as trading securities equivalents, tokenizing assets, taxing cryptocurrencies, etc.).

- Digital Divide: Today's blockchain tools and smart contracts are heavily codedependent, which poses a risk for non-coders who are unable to validate the specified functionality of the code. In addition, the lack of sufficient public education on blockchain distorts our understanding of technology.

- Platform self-regulation: Not only centralized platforms, but also decentralized platforms such as blockchain face self-regulatory challenges in terms of stewardship, corrective action, data responsibility, user rights, and other areas.

- Technical implementation: Blockchain still faces many technical challenges such as cybersecurity, data reversibility, and code loopholes.

- Scalability and Sustainability: Depending on the type of consensus algorithm used, some blockchain scaling may be difficult or impractical due to both transaction speed limits and energy cost / sustainability. It is primarily related to fully distributed and public blockchains, and there are new consensus algorithms that increasingly address scalability and sustainability issues.

- Persistent Security: Encrypted data in an immutable blockchain will be vulnerable at some point in the future, as encryption technology will become more computationally powerful and will have a finite lifespan as decryption technology evolves.

- Systematization of Negative Social Impacts: Who is involved in the creation and enforcement of blockchain rules using blockchain technology and is given access to join the blockchain can further solidify monopoly or other social power inequality based on someone.

\section{An Evolving Legal EnVIRONMENT}

Although blockchain may require changes to the applicable legal environment, today's blockchain participants are still bound by existing rules depending on the application in question. As is often the case with innovative technologies, existing legal principles provide a framework for their use, despite the lack of specific laws and regulations. Therefore, the lack of specific provisions regarding new technologies does not constitute a legal void (Donepudi, 2014b).

In France, the legal definition of blockchain was recently Ministerial Ordinance No. 28 April 2016 2016-520 on Certificates of Deposit. This order secretly defines blockchain as a shared electronic recording system that allows the verification of specific transactions of securities exchanged on crowdfunding platforms ("Minibonds"), L223-12 of the French Financial and Financial Law. Amend the article. Article L.223-13 states that "the transfer of ownership of the Minibond results from the entry of a sale to the shared electronic recording system referred to in Article L.223-12, which is the section of the French Civil Code. Articles 1321 and 1322 ". Recording the issuance of a minibond on the blockchain is 
considered to constitute a written contract. This written contract is required if the transaction is not invalidated. Therefore, it binds a third party.

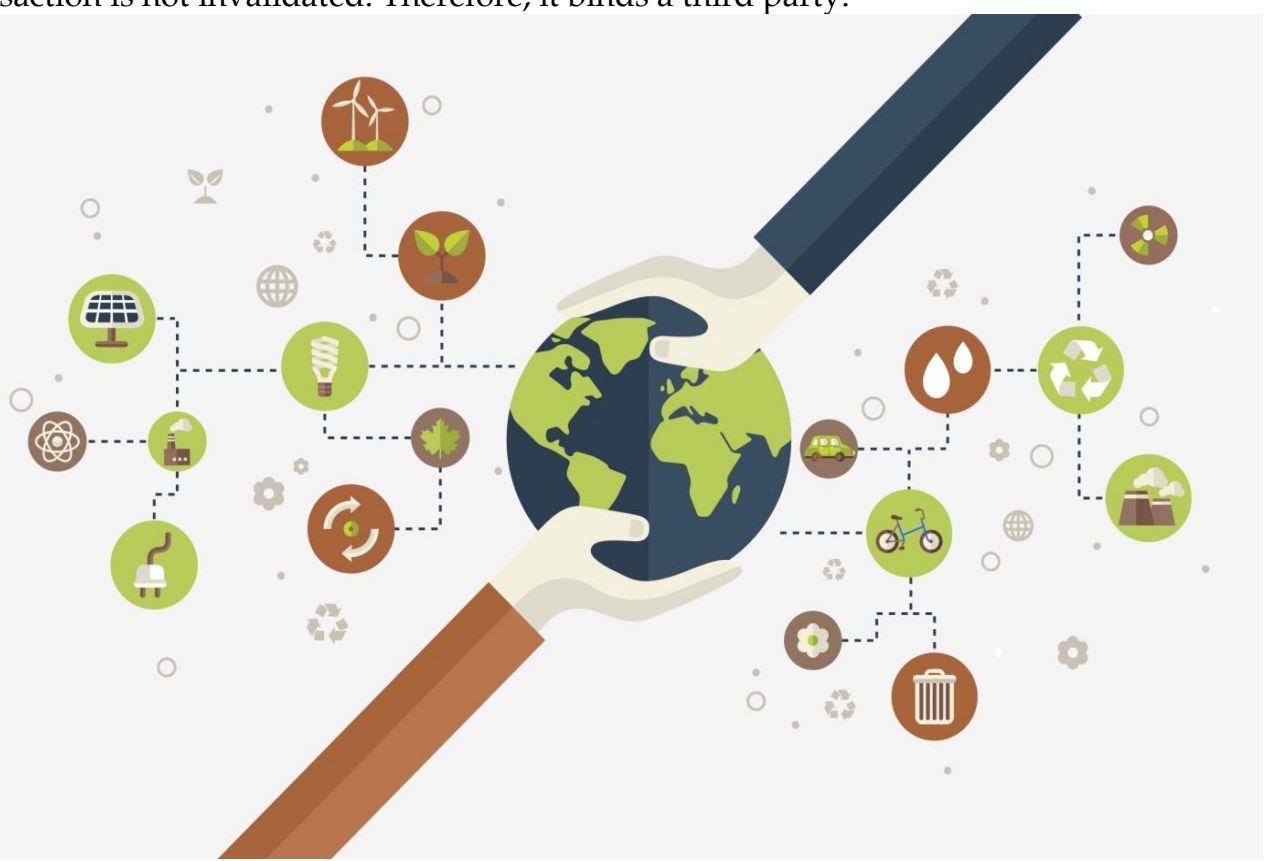

Legal issues arise especially when considering the legal validity of smart contracts that represent the decentralized automation capabilities of the blockchain. The term can be misleading because smart contracts are not as contracted as IT protocols that trigger the automatic execution of contract terms previously set by the parties (Donepudi, 2015). Theoretically, if the conditions required to establish an electronic contract are met, nothing precludes the contract contained in the smart contract that constitutes the written contract between the parties. Specifically, this means encoding the steps that provide proof of acceptance of a particular offer so that the parties are clearly identified so that the contract is legally valid. However, there are difficulties with the nature of technology rooted in immutability.

\section{REDUCTION IN KYC costs}

- In the insurance and banking industry and the public sector, the requirement to edit customer and stakeholder documents ("Know Your Customer", or KYC) is a costly and time-consuming process that can be fully transformed by distributed ledger technology.

- In reality, various documents are edited for specific customers in each organization without exchanging this information. Existing data centralization models make organizations particularly vulnerable. The number of cyberattacks is increasing, and the personal data of millions of customers is being stolen. Yahoo: One billion accounts were hacked in 2013 and 500 million accounts were hacked in 2014. eBay: 145 million cases were hacked in 2014. LinkedIn: 117 million in 2012. JP Morgan: Accounts for 76 million retail and seven million institutional customers hacked in 2014. In the blockchain, the data is not stored in the central database and the information is protected.

- Thanks to InterchainZ, a project born of a joint initiative of the PwC KYC Center of Excellence and $\mathrm{Z} \backslash$ Yen, a prototype of the KYC database was created using blockchain technology. 
- The idea is to store and encrypt customer data and validate any changes made (marriage, death, etc.) with anyone viewing the document. Customers are given a separate encryption key and choose whether to make it available to financial institutions.

- After that, financial institutions will have access to specific documents and data, and will be able to identify customers in a secure and reliable way. After that, the customer can immediately take out insurance or open an account.

- Without pooling data between insurance companies, banks, brokers, etc., this technology can already help significantly reduce the costs of the group and its subsidiaries (although cost savings along with the required investment spending). Should be considered).

- This is demonstrated by a partnership between IBM and Crédit Mutuel Arkéa. Crédit Mutuel Arkéa recently announced that it's completed its first blockchain project to enhance the bank's ability to verify customer identities.

- The result of this successful pilot is an operational prototype based on a private blockchain network that provides an overview of customer identities according to the KYC requirements of all group entities.Crédit Mutuel Arkéa recently announced that it has completed its first blockchain project to improve the bank's ability to verify customer identities.

- The result of this successful pilot is an operational prototype based on a private blockchain network that provides an overview of customer identities according to the KYC requirements of all group entities.

Table 1: Comparisons among public blockchain, consortium blockchain and private blockchain

\begin{tabular}{|l|l|l|l|}
\hline Property & Public blockchain & Consortium blockchain & Private blockchain \\
\hline $\begin{array}{l}\text { Consensus } \\
\text { determination }\end{array}$ & All miners & Selected set of nodes & One organization \\
\hline Read permission & Public & $\begin{array}{l}\text { Could be public or } \\
\text { restricted }\end{array}$ & $\begin{array}{l}\text { Could be public or } \\
\text { restricted }\end{array}$ \\
\hline Immutability & $\begin{array}{l}\text { Nearly impossible } \\
\text { to tamper }\end{array}$ & Could be tampered & Could be tampered \\
\hline Efficiency & Low & High & High \\
\hline Centralized & No & Partial & Yes \\
\hline Consensus process & Permissionless & Permissioned & Permissioned \\
\hline
\end{tabular}

Banks and insurance companies have different systems that individually manage customer identities for the different services they offer. As a result of this successful pilot, the Crédit Mutuel Arkéa group and IBM have put together various silos of customer data and unnecessarily duplicated information by creating a single cross-business KYC platform that notifies all banking processes. Reduce and request. Blockchain technology identifies and uses all valid existing data already stored within the bank's multiple recording systems, including data associated with loan applications, life assurance registrations, and opening bank accounts.

\section{LOWER RISK OF FRAUD AND THEFT}

Blockchain technology also helps organizations tackle fraud. As an example, Everledger, born of a startup accelerator program run by insurance company Allianz France, has developed a luxury certification system that uses a combination of private and public blockchain technologies. Everledger uses the blockchain to create a global registry of precious stones. Specifically, Everledger enters 40 properties (cut, color, transparency, etc.) for every recorded stone. It represents the 40 metadata components used to create a unique 
series number. This number is laser engraved on the stone and added to the associated blockchain. If the database contains enough data (more than 1 million diamonds were already recorded at the end of 2016), we cannot provide encrypted evidence that the seller owns the gem rights. If so, it will be very difficult to sell. Stones that do not have a serial number stamped on them or that are difficult to see are of substantial value. Everledger effectively contributes to the fight against theft and fraud by creating a global tamper-proof registry, with insurers paying an estimated US \$ 50 billion annually. An equivalent example can be found in the board and officer liability insurance market. It insures business leaders for actual or alleged errors that may be committed during the course of their duties, such as inaccurate financial statement disclosure or failure to comply with legal provisions. , Failed to pay salary, severance pay and taxes. Securing financial transactions (and the company's public financial statements) helps to increase transparency and thus mitigate the risk of markets with a theoretical total capacity of more than $€ 500$ million.

\section{AUTOMATION OF TASKS}

Thanks to blockchain technology, processes can be automated and made more secure by eliminating the need for specific instances of human input. A practical example of this is natural disaster insurance, which can be arranged using smart contracts that have been successfully piloted by the Allianz Group since June 2016. Group payment systems require two pieces of information that are built into the program.

- The event must have been declared a natural disaster.

- The location of the insured's event must correspond to an area recorded as having been hit by a natural disaster.

Table 2: Typical Consensus Algorithms Comparison

\begin{tabular}{|l|l|l|l|l|l|l|}
\hline Property & PoW & PoS & PBFT & DPOS & Ripple & Tendermint \\
\hline $\begin{array}{l}\text { Node } \\
\text { identity } \\
\text { manageme } \\
\text { nt }\end{array}$ & open & open & $\begin{array}{l}\text { Permis } \\
\text { sioned }\end{array}$ & open & open & permissioned \\
\hline $\begin{array}{l}\text { Energy } \\
\text { saving }\end{array}$ & no & partial & Yes & partial & Yes & Yes \\
\hline $\begin{array}{l}\text { Tolerated } \\
\text { power of } \\
\text { adversary }\end{array}$ & $\begin{array}{l}<25 \% \\
\text { computing } \\
\text { power }\end{array}$ & $\begin{array}{l}<51 \% \\
\text { stake }\end{array}$ & $\begin{array}{l}<33.3 \% \\
\text { faulty } \\
\text { replicas }\end{array}$ & $\begin{array}{l}<51 \% \\
\text { validat } \\
\text { ors }\end{array}$ & $\begin{array}{l}<20 \% \\
\text { faulty nodes } \\
\text { in UNL }\end{array}$ & $\begin{array}{l}<33.3 \% \\
\text { byzantine } \\
\text { voting power }\end{array}$ \\
\hline Example & Bitcoin & Peercoin & $\begin{array}{l}\text { Hyperled } \\
\text { ger Fabric }\end{array}$ & $\begin{array}{l}\text { Bitshar } \\
\text { es }\end{array}$ & Ripple & Tendermint \\
\hline
\end{tabular}

The purpose is to repeat Storm Xynthia (February 2010), where most victims no longer possess the necessary documents to file a claim and had to wait more than a year to receive insurance claims. Is to avoid. Not only are these types of incidents costly and protracted, but they also damage the insurer's reputation and make customers wary of the insurance system. When the Allianz Group uses a system based on reinsurance smart contracts ("natural disaster swaps"), it improves the way claims are handled while reducing human input (because the contracts are automated). The code automatically executes all eligible disaster insurance policies when an event occurs that meets the predefined conditions. This code also activates insurance payments directly without the customer having to submit the required documents. However, the principle of prohibiting unjust enrichment of claimants in the event of an insurance accident will continue to apply. 


\section{BETTER PRICING}

Recent advances in pricing show how static and dynamic behavioral factors affect risk, and insurers are gradually trying to capture these behaviors. Behavioral information is compiled by the connected device and exchanged on the blockchain, so prices are almost always adjusted and optimized based on the actual information reported in real time by the blockchain. This is still a very innovative area and still requires significant analysis and research. Regardless of blockchain technology, the insurance industry is inevitably evolving, with dynamic pricing and dynamic and flexible personalization due to voluntary data sharing and the ability to continuously assess customer behavior and risk profiles. Insurance products and risk management are realized. Blockchain-based dynamic pricing is clearly not in the foreseeable future, but the impact of technology on pricing can be felt sooner. The underwriting, pricing, and billing management processes are often faster and more efficient, for instance, by deploying rules within smart contracts. This in itself affects the competitiveness of the solutions we offer.

\section{EMERGENCE OF NeW MARKETS}

Blockchain technology will allow the development or expansion of new insurance lines and the entry into emerging markets. Today, especially in Africa, Asia and South America, $40 \%$ of the world's population do not have bank accounts or insurance. Blockchain technology allows insurers to develop personalized products and services more quickly and enhance their insurance offerings. For example, one of the trends in travel insurance is to pay insurance in real time in the event of a covered claim. This is a value proposition developed by Berkshire Hathaway Label Protection, for example. In this position, the insurer connects its system to the airline's system (for example, to get information about flight delays and cancellations) and identifies the affected customers of the flight in question in the customer database (Donepudi, 2014a). It means you need to. This proactively opens claims that give customers the right to make quick payments. Blockchain technology allows you to manage this value proposition in a fully automated way, reducing costs. InsurETH is predicated on Ethereum's smart contracts and therefore the results of the hackathon launched in 2015, and is positioned in just this sort of market. In this case, blockchain technology enables fast-paced development of new services linked to a particular product range. Blockchain also speeds up the development of personal insurance by combining data from contracts, invoices and customer documents in general.

\section{Applications of Blockchain In the Music Industry}

The growth of the Internet and the availability of numerous streaming services over the Internet have made a big difference in the music industry over the last decade. It affects everyone in the music industry, including artists, labels, publishers, songwriters and streaming service providers. The process of determining music royalties has always been complicated, but the rise of the Internet has made it even more complex, requiring transparency in payments by artists and songwriters. This is where blockchain can play a task by maintaining a comprehensive and accurate distributed database of music rights ownership information publicly ledgers. Additionally to the rights ownership information, you'll increase the database the royalty split for every work determined by the "smart contract". "Smart contracts" define relationships between different stakeholders (addresses) and automate their interactions. 


\section{DeCENTRALIZEd IoT}

IOT is becoming an increasingly popular technology in both consumer and business areas. Most IOT platforms are based on a centralized model in which a broker or hub controls the interaction between devices, but this approach is practical in many scenarios where devices need to exchange data autonomously. Is no longer. This particular requirement has led to efforts towards a decentralized IoT platform. Blockchain technology facilitates the implementation of distributed IoT platforms such as secure and reliable data exchange and records management. In such an architecture, the blockchain acts as a general ledger and maintains a reliable record of all messages exchanged between smart devices in a distributed IoT topology. IBM has partnered with Samsung to develop the platform ADEPT (Autonomous Decentralized Peer to Peer Telemetry) to build a distributed network of devices (Distributed Internet of Things (IOT)) using the design elements that underlie Bitcoin. ADEPT uses three protocols on the platform: Ethereum (smart contract), BitTorrent (file sharing) and TeleHash (peer-to-peer messaging).

\section{REFERENCES}

Ahram, Tareq, etc. "Blockchain Technology Innovation." Technology \& Engineering Management Conference (TEMSCON), 2017 IEEE. IEEE, 2017.

Ammous, Saifedean. "Blockchain Technology: What Does It Use for?" (2016).

Atzori, Marcella. "Blockchain Technology and Decentralized Governance: Is a State Still Needed?" (2015).

Condos, James, William H. Sorel, Susan L. Dnegan. "Blockchain Technology: Opportunities and Risks," Vermont, January 15, 2016.

Crossy, M., Pattanayak, P., Verma, S. , And Kalyanaraman, V. (2016). Blockchain TechNology: Beyond Bitcoin. Applied Innova-Tion,

Defilippi, Primavera, Samel Hassan. "Blockchain technology as a regulatory technology: from code to code." ArXiv Preprint arXiv: 1801.02507 (2018).

Donepudi, P. K. (2014a). Technology Growth in Shipping Industry: An Overview. American Journal of Trade and Policy, 1(3), 137-142. https://doi.org/10.18034/ajtp.v1i3.503

Donepudi, P. K. (2014b). Voice Search Technology: An Overview. Engineering International, 2(2), 91-102. https:/ / doi.org/10.18034/ei.v2i2.502

Donepudi, P. K. (2015). Crossing Point of Artificial Intelligence in Cybersecurity. American Journal of Trade and Policy, 2(3), 121-128. https://doi.org/10.18034/ajtp.v2i3.493

Kashan, Christian. "Hyper Leisure Blockchain Fabric Architecture". Workshop on decentralized cryptocurrencies and consensus ledgers. Volume 310.2016.

Marinova, Katya, Andrea Spark. "Market design using blockchain technology". (2017).

Nguyen, Quoc Khan. "Blockchain-Financial Technology for Sustainable Development in the Future." Green Technology and Sustainable Development (GTSD), International Conference. IEEE, 2016.

Nofer, Michael, etc. "Blockchain" Business \& Information Systems Engineering 59.3 (2017): 183-187

Pilkington, Mark. "11 Blockchain Technol-Ogy: Principles and Applications". Re-searching the Handbook on Digital Conversion-Mations (2016) 
Pilkington, Mark. "Blockchain Technology: Principles and Applications. Handbook of Research on Digital Conversion, f. Edited by Xavierolleros and majlindazhegu" (2016).

Zheng, Zibin, etc. "Overview of Blockchain Technology: Architecture, Consensus, and Future Trends" Bigdata Congress, 2017 Ieee International Congress On. Ieee, 2017

$--0--$ 